\title{
Editorial Reminders
}

The beginning of the year is a good time to refresh our memory on a few topics, and to mention a few other items that may be new to some of you.

PACS: The PACS classification scheme developed by the American Institute of Physics is no longer maintained, and its most recent version dates back to 2010. APS is working on a new classification scheme for physics, PhySH_Physics Subject Headings, that will replace PACS and is currently being beta tested. We encourage you to use it and give us feedback.

Manuscript length: Since 2011 Letters are no longer limited to four pages but rather to a maximum word count, currently 3750 words, not including abstract, authors list and affiliations, and references. Comments are limited to 750 words. Information on how to evaluate manuscript length is available at http://journals.aps.org/authors/length-guide.

Supplemental material (SM): SM should be limited to information that will be useful to a subset of readers, but that is not essential to the comprehension of the main results of the Letter. The key point is that the Letter must be self-contained and convincing to a wide readership without reference to the SM. Please keep in mind that failure of a manuscript to achieve this is sufficient reason for rejection. See http://journals.aps.org/authors/supplemental-materials-journals for more details.

Joint submissions: We encourage joint submission of a Letter and an accompanying regular article in Physical Review. This should be considered if the additional material is of sufficient importance and completeness that it can be turned into a regular article, perhaps geared to a narrower expert readership. We then vet the two submissions coherently and may coordinate their publication.

Justification: The "why PRL" justifications accompanying submissions are not always of the best quality. We hope to receive a substantive yet succinct explanation of why a paper is suitable for PRL, as opposed to some other journal. Ideally it should result from a thoughtful consideration of the specific PRL acceptance criteria listed in http://journals.aps.org/prl/about. Justifications that largely just restate one or more of our criteria are not particularly useful. Authors may want to ask themselves if, based on these criteria, they would truly recommend publication of the paper if they were its referees.

Highlights: Finally, we would like to remind you about Editors' Suggestions and the online publication Physics. PRL Editors' Suggestions highlight about one Letter in six, due to its particular importance, innovation, and broad appeal. Suggestions are downloaded twice as often as the average Letter, and are covered in the press substantially more often. If they were collected in a separate publication, they would have an Impact Factor of 13. Physics features news and commentary about papers in all of the APS journals, many from PRL. Viewpoints (by experts), Focus stories (by journalists), and Synopses (by editors) aim to broadly inform about new research with explanations that don't rely on jargon and technical detail.

Please visit Physics on a regular basis, sign up for the Physics email alerts if you haven't already, and take note of our highlighted Letters!

Pierre Meystre

Published 22 February 2016

Editor

DOI: 10.1103/PhysRevLett.116.080001 